\title{
MESOSCOPIC PHENOMENA IN MICROSTRUCTURES OF IV-VI EPILAYERS*
}

\author{
G. Grabecki, T. Dietl, W. Plesiewicz, A. Lenard, T. Skośkiewicz
}

Institute of Physics, Polish Academy of Sciences

Al. Lotników 32/46, 02-668 Warszawa, Poland

E. Kamińska, A. Piotrowska, E. Papis

Institute of Electron Technology, Al. Lotników 32/46, 02-668 Warszawa, Poland

\section{N. Frank and G. BaUer}

Institute für Halbleiterphysik, Johannes Kepler Universität Linz, 4040 Linz, Austria

Magnetoresistance measurements of photolithographically patterned $\mathrm{PbSe}$ and $\mathrm{Pb}_{1-x} \mathrm{Mn}_{x}$ Se microstructures were performed. Reproducible magnetoconductance fluctuations with the amplitude increasing with decreasing temperature were observed. Unexpectedly, these fluctuations contain a component periodic in the magnetic field, and their magnitude is greater than that expected from the current theory of the universal conductance fluctuations. Possible explanations are discussed.

PACS numbers: $71.55 . \mathrm{Jv}$

It is generally accepted by now that because of Aharonov-Bohm interference of scattered waves, the conductance of any disordered conductor should exhibit aperiodic fluctuations as a function of the magnetic field. The mean amplitude of these fluctuations increases with decreasing length $L$ of the sample and starts to be universal, i.e., independent of the material that the sample is made of, $\Delta G \approx 0.5 e^{2} / h$, once $L$ becomes smaller than the phase breaking length $L_{\phi}(T)[1,2]$. Accordingly, the phenomenon is known under the name of universal conductance fluctuations (UCF). Indeed, a series of beautiful millikelvin experiments with nanostructures of nonmagnetic metals has fully confirmed the theoretical predictions [3]. In the case of semiconductors, however, the situation is less clear. In particular, the presence of a component periodic in the magnetic field has been detected in microstructures of $n^{+} \mathrm{GaAs}$ [4], $\mathrm{Hg}_{1-x-y} \mathrm{Cd}_{y} \mathrm{Mn}_{x} \mathrm{Te}$ bicrystals [5], GaAs/AlGaAs heterostructures [6]. Besides, recent studies of magnetic metallic [7] and $\mathrm{Hg}_{1-x-y} \mathrm{Cd}_{y} \mathrm{Mn}_{x}$ Te microstructures [8] have contradicted those

*The work in Poland was supported by the grant No. 204669101 of the State Committee for Scientific Research (Republic of Poland). 
theoretical suggestions [9] which concern the time dependence of the fluctuation pattern in the spin-glass phase. We performed magnetotransport measurements of photolithographically patterned microstructures of $\mathrm{Pb}_{1-x} \mathrm{Mn}_{x} \mathrm{Se}(0 \leq x \leq 2 \%)$. The starting material consisted of $2 \div 3 \mu \mathrm{m}$ thick $n$-type epilayers grown by molecular beam epitaxy on a $\mathrm{BaF}_{2}$ substrate. Microstructures in the form of Hall bars with the linewidth $2-100 \mu \mathrm{m}$ and different geometrical factors, $L / W=0.33 \div 6$, were prepared by photolithography and Ar ion milling [10]. The measurements were performed in the magnetic field range up to $120 \mathrm{kOe}$, at temperatures between $50 \mathrm{mK}$ and $40 \mathrm{~K}$. To ensure the linear response, the current was kept at the level of $300 \mathrm{nA}$.

Characterization of the structures by measurements of the conductivity and the weak-field Hall effect at $2 \mathrm{~K}$ shows that the samples are $n$-type with the electron concentration (1-2) $\times 10^{17} \mathrm{~cm}^{-3}$, and the mobility summarized in Table. Inspection of this table points to certain degradation of the carrier mobility when decreasing the linewidth, suggesting the formation of etching-induced defects. In particular, a change of sign of the Hall resistance in high magnetic fields, which we observed in some microstructures, indicates that the ion bombardment creates acceptor centers. Furthermore, we noticed a gradual decrease in the resistivity, up to the factor of four between $1 \mathrm{~K}$ and $50 \mathrm{mK}$. We assign this phenomenon to the presence of some superconducting $\mathrm{Pb}$ inclusions, and a long penetration length of the Cooper pairs in our high mobility material at low temperatures. The critical field for this superconductivity is as low as 50 Oe.

The most surprising result of our study is the detection of unusual reproducible conductance fluctuations as a function of the magnetic field in $\mathrm{PbSe}$ and $\mathrm{Pb}_{1-x} \mathrm{Mn}_{x}$ Se. The magnitude of these fluctuations attains about $0.1 \%$ of the resistance values which, in turn, increase by one order of magnitude when the

TABLE

Electron mobility and the fluctuation amplitude in different microstructures.

\begin{tabular}{c|c|c|c}
\hline \hline Layer No. & $\begin{array}{c}\text { Structure dimensions } \\
\text { Mn cont. }\end{array}$ & $\begin{array}{c}\text { Hall mobility } \\
{[\mu \mathrm{m}]}\end{array}$ & $\begin{array}{c}\mathrm{rms}(\Delta G) \\
{\left[\mathrm{m}^{2} / \mathrm{Vs}\right]}\end{array}$ \\
\hline PS98 & $200 \times 100 \times 2.75$ & 29.4 & 0.67 \\
PbSe & $24 \times 4 \times 2.75$ & 5.76 & 0.77 \\
& $6 \times 20 \times 2.75$ & 9.2 & 1.47 \\
& $14 \times 20 \times 2.75$ & 4.8 & 2.10 \\
\hline PMS7 & $200 \times 100 \times 2.0$ & 7.8 & 0.044 \\
PbMnSe & $24 \times 4 \times 2.0$ & 3.07 & 0.036 \\
$x=0.6 \%$ & $18 \times 2 \times 2.0$ & 2.08 & 0.045 \\
& $6 \times 2 \times 2.0$ & 2.3 & 0.18 \\
& $2 \times 2 \times 2.0$ & 2.2 & 1.97 \\
\hline PMS95 & $24 \times 4 \times 2.75$ & 2.05 & 0.20 \\
PbMnSe & $12 \times 2 \times 2.75$ & 0.046 & 0.15 \\
$x=1.74 \%$ & $6 \times 2 \times 2.75$ & 0.046 & 0.16
\end{tabular}




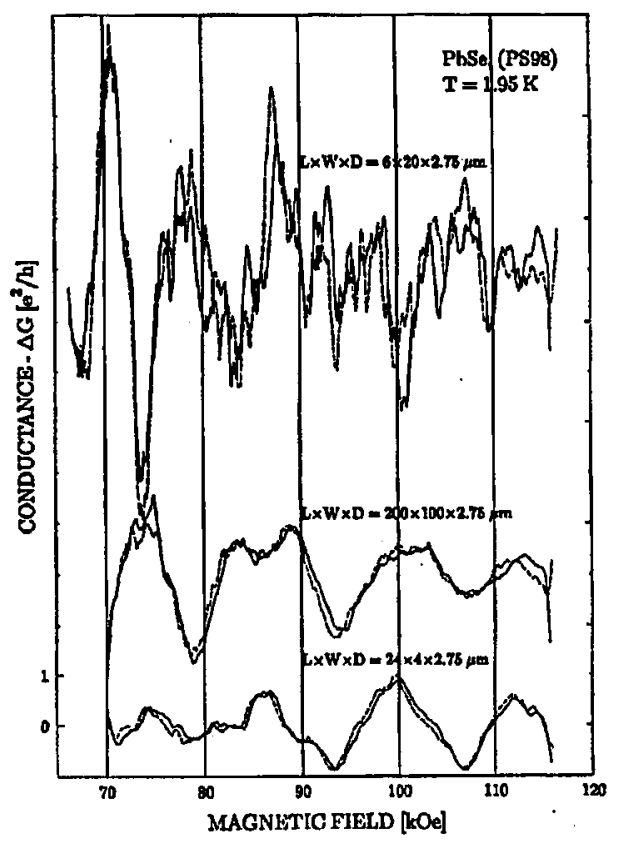

Fig. 1. Field-induced conductance fluctuations as measured for two sweep directions (solid and dashed lines, respectively) for $\mathrm{PbSe}$ microstructures of various geometrical dimensions. The magnetic field was perpendicular to the epilayer plane.

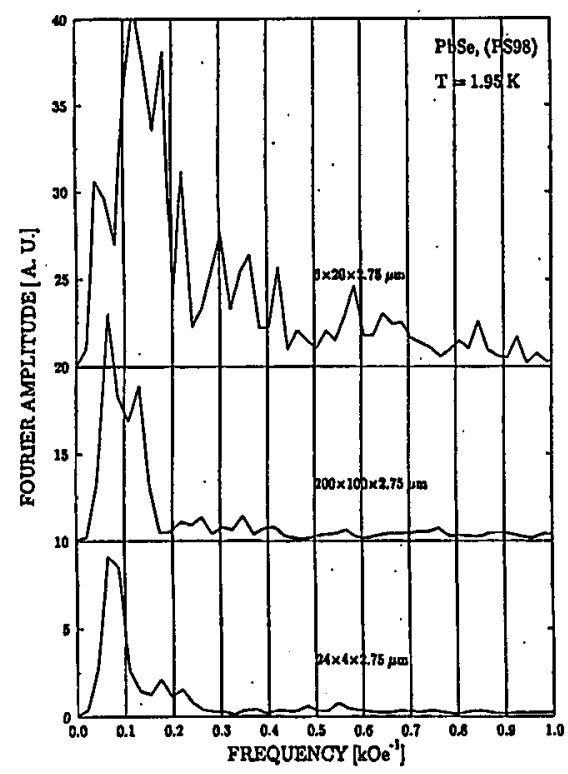

Fig. 2. Fourier transform of experimental traces presented in Fig. 1 for PbSe microstructures. 
magnetic field increases up to $120 \mathrm{kOe}$. As shown in Figs. 1 and 2, where the magnetoresistance and its Fourier transform for three structures of $\mathrm{PbSe}$ are presented, the fluctuations are quasi-periodic in the magnetic field. Moreover, the magnitude of the fluctuations is greater than $0.5 e^{2} / h$, independently of the size of the structures. Furthermore, they are rather insensitive to the temperature changes - they start to decrease above $1 \mathrm{~K}$, but they are still visible at $30 \mathrm{~K}$. Similar fluctuations but, as depicted in Table, of a little smaller amplitude, were found in $\mathrm{Pb}_{1-x} \mathrm{Mn}_{x} \mathrm{Se}$.

We propose two different mechanisms which may account for the reproducible fluctuations that are quasi-periodic in the magnetic field:

(i) These fluctuations may originate from the field-induced shift of electric subbands. Indeed, such dimensional quantization of the electron spectrum could occur in our epilayers as their thickness is comparable to the mean free path of the electrons. An attempt is underway to detect similar fluctuations in epilayers from other materials with comparable mobility.

(ii) Quasi-periodic fluctuations in singly connected geometry could be caused by Aharonov-Bohm interference of self-intersecting trajectories provided that the scattering potential contains a component periodic in space. The period of $10 \mathrm{kOe}$ that we observe would then correspond to the radius of the Aharonov-Bohm loops of $100 \AA$. We suggest that misfit dislocations or space-correlated resonant donors might result in quasi-periodic network of electron trajectories. This hypothesis merits further experiments in systems where space correlation between scattering centers can be affected by external perturbations.

\section{References}

[1] B.L. Al'thsuler, B.I. Skhlowskii, Zh. Eksp. Teor. Fiz. 91, 220 (1986).

[2] P.A. Lee, A. Douglas Stone, H. Fukuyama, Phys. Rev. B 35, 1039 (1987).

[3] S. Washburn, R.A. Webb, Rep. Prog. Phys. 55, 1311 (1992).

[4] A. Ramon, M. Heiblum, H. Shrinkman, Semicond. Sci. Technol. 8, 2175 (1993).

[5] T. Dietl, G. Grabecki, J. Jaroszyński, Semicond. Sci. Technol. 8, S141 (1993).

[6] J. Wróbel, G. Grabecki, F. Kuchar, T. Dietl, K. Ismail, K.Y. Lee, H. Nickel, W. Schlapp, to be published.

[7] C. Van Haesendonck, H. Vloeberghs, H. Bruynseraede, R. Jonckheere, in: Nanostructures Physics and Fabrication, Eds. M.A. Reed, W.P. Kirk, Academic Press, Boston 1989, p. 467; A. Benoit, S. Washburn, R.A. Webb, D. Mailly, L. Dumoulin, in: Anderson Localization, Eds. T. Ando, H. Fukuyama, Springer, Berlin 1988, p. 346; P.G.N. de Vegvar, L.P. Levy, T.A. Fulton, Phys. Rev. Lett. 66, 2380 (1991).

[8] G. Grabecki, T. Dietl, W. Plesiewicz, A. Lenard, T. Skośkiewicz, E. Kamińska, A. Piotrowska, Physica B 194-196, 1107 (1994).

[9] B.L. Al'thsuler, B.Z. Spivak, Pis'ma Zh. Eksp. Teor. Fiz. 42, 363 (1985); S. Feng, A.J. Bray, P. Lee, M.A. Moore, Phys. Rev. B 36, 5624 (1987); Marek Cieplak, B.R. Bułka, T. Dietl, Phys. Rev. B 44, 12337 (1991).

[10] G. Grabecki, T. Dietl, Marek Cieplak, A. Lenard, W. Plesiewicz, T. Skośkiewicz, E. Kamińska, A. Piotrowska, R. Żarecka, G. Springholz, G. Bauer, Acta Phys. Pol. A 84, 781 (1993). 